\title{
AN ALMOST-ORTHOGONALITY PRINCIPLE WITH APPLICATIONS TO MAXIMAL FUNCTIONS ASSOCIATED TO CONVEX BODIES
}

\author{
BY ANTHONY CARBERY 1
}

1. Introduction. Let $B$ be a convex body in $R^{n}$, normalised to have volume one. Let $M$ be the centred Hardy-Littlewood maximal function defined with respect to $B$, i.e.

$$
M f(x)=\sup _{t} t^{-n} \int_{t B}|f(x-y)| d y .
$$

Let $\tilde{M}$ be the lacunary maximal operator,

$$
\tilde{M} f(x)=\sup _{k} 2^{-k n} \int_{2^{k} B}|f(x-y)| d y .
$$

Considerable interest has recently been shown in the behaviour of these operators for large $n$, see $[\mathbf{1}, \mathbf{2}, \mathbf{8}, \mathbf{9}, \mathbf{1 0}]$. When $B$ is the ball, Stein has shown [8] that $M$ is bounded on $L^{p}\left(R^{n}\right), 1<p \leq \infty$, with a constant $C_{p}$ depending only on $p$, and not on $n$; Stein and Strömberg [10] have shown that for $p$ larger than 1 , the $L^{p}$ operator norm of $M$ is at most linear in the dimension. More recently Bourgain has proved that the $L^{2}$ operator norm of $M$ is bounded by an absolute constant independent of the body and the dimension [1]. It is the purpose of this note to extend this result to $p>3 / 2$, and to all $p>1$ if we instead consider $\tilde{M}$.

THEOREM 1. (i) Let $p>3 / 2$. Then there exists a constant $C_{p}$, depending only on $p$ and not on $B$ or $n$, such that $\|M f\|_{p} \leq C_{p}\|f\|_{p}$.

(ii) Let $p>1$. Then there exists a constant $D_{p}$, depending only on $p$ and not on $B$ or $n$, such that $\|\tilde{M} f\|_{p} \leq D_{p}\|f\|_{p}$.

It has recently been brought to the author's attention that part (i) of the theorem has been proved by Bourgain ${ }^{2}$ in the special case that $B$ is the cube [2]. Here we show that Theorem 1 in fact follows from Bourgain's previous analysis together with a general almost-orthogonality principle for maximal functions, Theorem 2. A weaker version of this principle appears in [6], where it is also applied to various operators including maximal functions and Hilbert transforms along curves. A similar principle due to Michael Christ appears in $[\mathbf{4}]$.

Full details of the proofs, together with further applications, will appear elsewhere.

Received by the editors September 13, 1985 .

1980 Mathematics Subject Classification (1985 Revision). Primary 42B15, 42B25.

${ }^{1}$ Partially supported by an NFS grant.

${ }^{2}$ NOTE ADDED IN PROOF. Theorem 1 has been proved in full independently by J. Bourgain. 
2. An almost-orthogonality principle. Let $T_{j v}, j \in Z, v \in S$, be a family of linear operators (multiplier operato. in our application). Here $S$ is any indexing set. Suppose there are linear operators $R_{j}(j \in Z)$ such that $\sum R_{j}=I$. Consider the maximal operator $T_{*} f(x)=\sup _{j v}\left|T_{j v} f(x)\right|$.

DEFINITION. (i) $T_{*}$ is weakly bounded (with respect to $R_{j}$ ) on $L^{q}$ if

$$
\sup _{k}\left\|\sup _{j v}\left|T_{j v} R_{j+k} f\right|\right\|_{q} \leq C\|f\|_{q} .
$$

(ii) $T_{*}$ is strongly bounded on $L^{q}$ if for some sequence $a_{k}$ satisfying $\sum a_{k}^{t}<\infty, 0<t \leq 1$,

$$
\left\|\sup _{j v}\left|T_{j v} R_{j+k} f\right|\right\|_{q} \leq a_{k}\|f\|_{q} .
$$

With this definition it is clear that strong boundedness on $L^{q}$ implies boundedness on $L^{q}$, which implies weak boundedness on $L^{q}$, provided of course that the $R_{j}$ 's are uniformly bounded on $L^{q}$. Moreover, if $T_{*}$ is strongly bounded on some $L^{q_{0}}$ space and weakly bounded on $L^{q_{1}}$, then $T_{*}$ is bounded on $L^{q}$ for all $q$ strictly between $q_{0}$ and $q_{1}$.

DEFINITION. A family $T_{j v}$ of linear operators is essentially positive if there exist linear operators $S_{j v}$ and $U_{j v}$, with $S_{j v} \geq 0, U_{j v} \geq 0, S_{*}$ bounded on $L^{r}$, $1<r \leq \infty$, and such that $T_{j v}=U_{j v}-S_{j v}$.

THEOREM 2. Let $1 \leq p<2$, and let $T_{j v}$ be an essentially positive family of linear operators. Suppose there exists a $q \neq p$ (we shall assume $q>p$ ) such that $T_{*}$ is strongly bounded on $L^{q}$, and suppose there exists an $\varepsilon>0$ such that $\sup _{j}\left\|\sup _{v}\left|T_{j v} f\right|\right\|_{r} \leq C_{r}\|f\|_{r}$ and $\left\|\left(\sum\left|R_{j} f\right|^{2}\right)^{1 / 2}\right\|_{r} \leq C_{r}\|f\|_{r}$ for $r$ in $(p, p+\varepsilon)$. Then $T_{*}$ is bounded on $L^{r}$ for all $r$ in $(p, q]$.

REMARK. There is a similar but simpler principle when $p \geq 2$, whose statement and proof we omit.

PROOF. We first assume that for all but finitely many $j, T_{j v}=0$ for all $v$, and we shall obtain a bound for $T_{*}$ independent of this finite number $N$. So, fixing an $r$ with $p<r<q$, we may assume that $\left\|T_{*} f\right\|_{r} \leq A(N)\|f\|_{r}$.

We consider first inequalities of the form

$$
\|\| \sup _{v}\left|T_{j v} g_{j}\right|\left\|_{l^{s}}\right\|_{L^{t}} \leq C_{s, t}\|\| g_{j}\left\|_{l^{s}}\right\|_{L^{t}} .
$$

By assumption, $(*)$ holds for $s=t$ in $(p, p+\varepsilon)$. It also holds with $t=r$ and $s=\infty$, with constant $B(N)$ depending on $A(N)$ since $U_{j v}$ and $S_{j v}$ are positive. Thus by interpolation there exists an $\tilde{r}, p<\tilde{r}<r$, such that (*) 
holds for $t=\tilde{r}$ and $s=2$. Now,

$$
\begin{aligned}
\left\|\sup _{j v}\left|T_{j v} R_{j+k} f\right|\right\|_{\tilde{r}} & \leq\left\|\left(\sum_{j} \sup _{v}\left|T_{j v} R_{j+k} f\right|^{2}\right)^{1 / 2}\right\|_{\tilde{r}} \\
& \leq D(N)\left\|\left(\sum_{j}\left|R_{j+k} f\right|^{2}\right)^{1 / 2}\right\|_{\tilde{r}} \\
& \leq C_{r} D(N)\|f\|_{\tilde{r}} .
\end{aligned}
$$

So $T_{*}$ is weakly bounded on $L^{\tilde{r}}$ and hence by the comment preceding the theorem it is bounded on $L^{r}$ with constant $E(N)$. However, keeping track of constants, we see that for some $0<t<1$ and some numbers $a$ and $b$, $E(N) \leq a+b A(N)^{t}$. Thus $A(N) \leq c$, for some $c$ independent of $N$, concluding the proof of the theorem.

3. An auxiliary proposition. We now specialise to operators of the form $\left(T_{j t} f\right)^{\wedge}(\xi)=m\left(2^{j} t \xi\right) f^{\wedge}(\xi)$ for $j \in Z$ and $1 \leq t \leq 2$. We wish to apply Theorem 2 in the case $q=2$, and so we need simple criteria for determining when a maximal operator is bounded on $L^{2}$, and when a maximal operator of the form $\sup _{1 \leq t \leq 2}\left|K_{t} * f\right|$ is bounded on $L^{p}$.

Proposition. Let $K^{\wedge}=m \in L^{\infty}$. Then

(i) $\left\|\sup _{0<t<\infty}\left|K_{t} * f\right|\right\|_{2} \leq C\|f\|_{2}$ if for some $\alpha>\frac{1}{2}$ we have

$$
\sup _{w \in S^{n-1}}\left(\int_{0}^{\infty}\left|u^{\alpha+1}(d / d u)^{\alpha}\left[u^{-1} m(u w)\right]\right|^{2} d u / u\right)^{1 / 2}<\infty
$$

(ii) $\left\|\sup _{1 \leq t \leq 2}\left|K_{t} * f\right|\right\|_{p} \leq C\|f\|_{p}$ if for some $\alpha>1 / p($ or $\alpha=1$ if $p=1)$ both $m$ and $(\xi \cdot \nabla)^{\alpha} m$ are $L^{p}$ multipliers.

REMARKS. (i) Here, $(d / d u)^{\alpha}$ is the fractional differentiation operator defined for example in [3], and

$$
(\xi \cdot \nabla)^{\alpha} m(\xi)=\left.(d / d u)^{\alpha} m(u \xi)\right|_{u=1}=\int(2 \pi i x \cdot \xi)^{\alpha} K(x) e^{2 \pi i x \cdot \xi} d x .
$$

(ii) When $m$ is radial, part (i) of the proposition is in [3]. Other similar criteria for $L^{2}$ boundedness of maximal operators appear in [1, 5 and 7 .

ProOF. Write

$$
\frac{m(t \xi)}{t}=C_{\alpha} \int_{0}^{\infty}(u-t)_{+}^{\alpha-1}(d / d u)^{\alpha}[m(u \xi) / u] d u
$$

Therefore,

$$
\left|K_{t} * f\right| \leq C_{\alpha} \int_{0}^{\infty}(1-t / u)_{+}^{\alpha-1} t / u\left|P_{u}^{\alpha} f\right| d u / u
$$

where $\left(P_{u}^{\alpha} f\right)^{\wedge}(\xi)=u^{\alpha+1}(d / d u)^{\alpha}[m(u \xi) / u] f^{\wedge}(\xi)$. Thus, if $p=2$ and $\alpha>\frac{1}{2}$,

$$
\sup _{0<t<\infty}\left|K_{t} * f\right| \leq C_{\alpha}\left(\int_{0}^{\infty}\left|P_{u}^{\alpha} f\right|^{2} d u / u\right)^{1 / 2}
$$


and so

$$
\left\|\sup _{0<t<\infty}\left|K_{t} * f\right|\right\|_{2} \leq C_{\alpha}\|f\|_{2}
$$

if the hypothesis of part (i) is fulfilled. If $p \neq 2$ and $t \geq 1$,

$$
\begin{aligned}
\left|K_{t} * f\right| & \leq C_{\alpha}\left(\int_{1}^{\infty}\left|(1-t / u)_{+}^{\alpha-1} t / u\right|^{q} d u\right)^{1 / q}\left(\int_{1}^{\infty}\left|P_{u}^{\alpha} f\right|^{p} d u / u^{p}\right)^{1 / p} \\
& \leq C_{\alpha} t^{1 / q}\left(\int_{1}^{\infty}\left|P_{u}^{\alpha} f\right|^{p} d u / u^{p}\right)^{1 / p}, \quad \text { if } 1 / p+1 / q=1 \text { and } \alpha>1 / p .
\end{aligned}
$$

Hence

$$
\left\|\sup _{1 \leq t \leq 2}\left|K_{t} * f\right|\right\|_{p} \leq C_{\alpha}\left(\int_{1}^{\infty}\left\|P_{u}^{\alpha} f\right\|_{p}^{p} d u / u^{p}\right)^{1 / p} .
$$

But the $L^{p}$ operator norm of $P^{\alpha}$ is controlled by the $L^{p}$ multiplier norms of $m$ and $(\xi \cdot \nabla)^{\alpha} m$.

4. Proof of Theorem 1. At this point we shall assume that the reader is familiar with the contents of $[\mathbf{1}]$, where, amongst other things, Bourgain proves that there exist a number $L=L(B)$ and an $A \in \mathrm{SL}(n, R)$ such that if $K=\chi_{A(B)}$, then

(a) $\left|K^{\wedge}(\xi)\right| \leq C(|\xi| L)^{-1}$

(b) $\left|K^{\wedge}(\xi)-1\right| \leq C|\xi| L$,

(c) $\left|\xi \cdot \nabla K^{\wedge}(\xi)\right| \leq C$,

with $C$ an absolute constant.

We shall obtain a Littlewood-Paley decomposition of $R^{n}, I=\sum R_{j}$, such that $\left\|\left(\sum\left|R_{j} f\right|^{2}\right)^{1 / 2}\right\|_{p} \leq C_{p}\|f\|_{p}, 1<p \leq 2$, with $C_{p}$ independent of $n$, then observe that the essentially positive family of operators $\left(K-P_{L}\right)_{t>0}$ has maximal function strongly bounded on $L^{2}$ with respect to this decomposition, with constant independent of everything. Here $P$ is the Poisson kernel; it is of course true that $P_{*}$ is bounded on $L^{p}\left(R^{n}\right), 1<p \leq \infty$ with constant independent of $n$ (see [8]). Finally we show that $\left\|\sup _{1 \leq t \leq 2}\left|K_{t} * f\right|\right\|_{p} \leq$ $C_{p}\|f\|_{p}$, with $C_{p}$ independent of $B$ and $n$ if $p>3 / 2$, which will conclude the proof once we apply Theorem 2 ; for the case of $\tilde{M}$ this third step is not required since $\|K\|_{1}=1$.

Each of these steps is easy; for the first we merely take $R_{j}=P_{2^{j+1}}-P_{2^{j}}$; then $\left(\sum\left|R_{j} f\right|^{2}\right)^{1 / 2} \leq(\log 2)^{1 / 2} g_{1}(f)(x), g_{1}$ being the classical LittlewoodPaley function, which Stein has shown [9] to satisfy $\left\|g_{1}(f)\right\|_{p} \leq C_{p}\|f\|_{p}$, $1<p \leq 2$, with $C_{p}$ independent of $n$.

For the second step, one may apply part (i) of the proposition to each of the operators $\left(K-P_{L}\right) R_{k}, k \in Z$, using (a)-(c) to obtain $\left\|\left[K-P_{L}\right] R_{k *}\right\|_{2} \leq$ $C a_{k}\|f\|_{2}$, with $\sum a_{k}^{t}<\infty, 0<t \leq 1$. This is not exactly what being strongly bounded on $L^{2}$ means, but a slight modification of this argument will give precisely what we require.

Finally, observe that $K^{\wedge}$ has $L^{1}$ multiplier norm 1, and by (c) above $(\xi \cdot \nabla) K^{\wedge}$ has $L^{2}$ multiplier norm dominated by an absolute constant; after setting up the appropriate complex-analytic interpolation argument, one obtains that $(\xi \cdot \nabla)^{\alpha} K^{\wedge}$ has $L^{p}$ multiplier norm dominated by an absolute 
constant if $\alpha<2 / p^{\prime}, 0<\alpha<1,1<p<2$. An application of part (ii) of the proposition yields $\left\|\sup _{1 \leq t \leq 2}\left|K_{t} * f\right|\right\|_{p} \leq C_{p}\|f\|_{p}$, with $C_{p}$ depending only on $p$ if $1 / p<2 / p^{\prime}$, which is $p>3 / 2$.

5. Concluding remark. The reader will observe that only the last of the three steps does not work for all $p>1$; if the method is to succeed further, results of the form $(\xi \cdot \nabla) K^{\wedge}$ having $L^{p}$ multipler norm not depending on $B$ or $n, p \neq 2$, would be useful. Of course the $L^{1}$ multiplier norm of this operator is essentially $n$. Is it possible to do better than interpolation between $p=1$ and $p=2$ for this operator? Such results, if true, would give a new expression to the philosophy that, for large $n$, "most of the mass of a convex body is situated near its boundary".

\section{REFERENCES}

1. J. Bourgain, On high dimensional maximal functions associated to convex bodies (preprint).

2. Maximal functions in $R^{n}$ and cubes (preprint).

3. A. Carbery, Radial Fourier multipliers and associated maximal functions, Recent Progress in Fourier Analysis, North-Holland Math. Studies Vol. 111, 1985, 49-56.

4. __ Differentiation in lacunary directions and an extension of the Marcinkiewicz multiplier theorem (in preparation).

5. H. Dappa and W. Trebels, On maximal functions generated by Fourier multipliers (manuscript).

6. J. Duoandikoetxea and J. L. Rubio de Francia, Maximal and singular integral operators via Fourier transform estimates (preprint).

7. C. Sogge and E. M. Stein, Averages of functions over hypersurfaces in $R^{n}$ (preprint).

8. E. M. Stein, The development of square functions in the work of Antoni Zygmund, Bull. Amer. Math. Soc. (N.S.) 7 (1982), 359-376.

9. Soc. (N.S.) 9 (1983), 71-73.

10. E. M. Stein and J. O. Strömberg, Behavior of maximal functions in $R^{n}$ for large $n$, Ark Mat. 21 (1983), 259-269. 60637

Department of Mathematics, University of Chicago, Chicago, Illinois

Current address: Division of Mathematics, University of Sussex, Falmer, Brighton, Sussex BN1 9QH, England 
